\title{
Investigation of Effects of L-Theanine on PTSD-induced Changes in Rat Neurobehavior
}

\author{
Tomás Eduardo Ceremuga ${ }^{凶}$, Michael Bentley, Jeremiah Wolfe, Susan Baldwin, Thomas Onstott, Ken \\ Aytes, Bryan Ferrara, Mark Alleyn, Chris Fortner, George A. Ceremuga and Gina Padrón
}

\begin{abstract}
Post-traumatic stress disorder (PTSD) is a devastating neuropsychological disorder that may develop in response to traumatic experiences. Symptoms include anxiety, hypervigilance, memory deficits, and depression. We investigated L-Theanine on neurobehavioral effects in a PTSD rodent model. Evaluation of the effects of L-Theanine as a sole agent and in combination with midazolam on neurobehavior was analyzed using the Elevated Plus-Maze, Morris Water Maze, and Forced Swim Test. Statistical analysis consisted of comparison of PTSD symptoms in PTSD vs. non-stressed groups. Data regarding weight gain between the 40 control (non-stressed) and 40 PTSD (stressed) rats were significantly different $(p<0.001)$, where the control rats gained an average of 55.4 grams compared to 37.4 grams for the PTSD rats over the 10 post stress days. This research did not show statistical significance with single dose administration of L-Theanine or in combination with midazolam. However, the theoretic framework and
\end{abstract}

Received: 5 November 2014

Accepted revised version: 16 December 2014

Published online: 1 January 2015

(C) Ceremuga et al (2015)

Publisher: Horizon e-Publishing Group

\section{CITATION}

Ceremuga, T. E., M. Bentley, J. Wolfe, S. Baldwin, T. Onstott, K. Aytes, B. Ferrara, M. Alleyn, C. Fortner, G. A. Ceremuga, and G. Padrón. 2015. Investigation of Effects of L-Theanine on PTSD-induced Changes in Rat Neurobehavior. Plant Science Today 2: 11-20. doi: 10.14719/pst.2015.2.1.91

\section{AUTHOR'S AFFILIATION}

US Army Graduate Program in Anesthesia Nursing.

CORRESPONDENCE

$\triangle$ Dr. Tomás Eduardo Ceremuga, CRNA, AMEDDC\&S Nursing Science, ATTN: Tomás Eduardo Ceremuga, 3490 FORAGE RD; STE 112, FT SAM HOUSTON TX 78234-7585, Comm: (210) 833-0786, Fax: (210) 295-4858. Email: thomas.e.ceremuga.civ@mail.mil
Post-Traumatic Stress Disease Induction Model were validated based on this research. This study establishes a solid framework for future investigation of PTSD treatments. Future studies of L-Theanine and other herbal therapies may use an extended dosing period to obtain a steady state for the period of time needed to alter neurobiology.

Keywords: PTSD; Neurobehavior; Green Tea; L-Theanine

\section{Introduction}

Post-traumatic stress disorder (PTSD) is a devastating neuropsychological injury with increasing occurrence. It is estimated that $58 \%$ of the Iraq and Afghanistan veteran population demonstrate some PTSD-related symptoms (Servatius et al., 1995; Shiner et al., 2012). The psychological, physical, and emotional symptoms of PTSD are complex and debilitating. Social manifestations of PTSD are crippling. Interpersonal relations are often strained with conflict, social withdrawal and alienation, reduced intimacy, and impaired work performance. Furthermore, there are often somatic complaints of exhaustion, insomnia, headaches, exaggerated reflexes, hyperarousal, cardiovascular, gastrointestinal, and musculoskeletal disorders (Jankowsi, 2010). Intrusive thoughts, nightmares, avoidance of reminders of the traumatic event, and anxiety are also associated with PTSD (Ursano et al., 2009). A recent meta-analysis revealed memory impairment in subjects with PTSD is present regardless of the population studied or presence of physical injury (Brewin et al., 2007). Depression is a common comorbidity in PTSD, and may also contribute to the physical and social symptoms associated with PTSD (Jankowsi, 2010). An optimal treatment modality has not been found, hence, current treatment for PTSD is multimodal, complicated, and inconsistent. The continuum of treatment ranges from family therapy, psychotherapy, cognitive behavioral therapy, and pharmacotherapy. 
Recently, the green tea component, L-Theanine, has gained popularity for the self-treatment of anxiety ( $\mathrm{Lu}$ et al., 2004; Heese et al., 2009). Green tea, Camelia sinensis L., has been a popular beverage in many cultures for thousands of years. In 2009, the Los Angeles Times discussed the use of L-Theanine in popular drinks such as SoBe Lifewater, Vitamin Water, and Gatorade (Roan, 2009). It has long been postulated that green tea has multiple health benefits such as anxiolysis and relaxation. Over the last 50 years, these effects have been scientifically linked to L-Theanine (Juneja et al., 1999).

L-Theanine, a derivative of glutamate, is absorbed in the small intestine, metabolized by the kidney, and crosses the blood-brain barrier (Eschenauer and Sweet, 2006). The effects of L-Theanine on the brain are not fully understood. These effects include the following: (1) inhibition of glutamate reuptake, (2) increase in gamma-amino butyric acid (GABA), (3) increase in dopamine release in the striatum, and (4) increase in serotonin levels (Charney et al., 2001). Additionally, modulation of glutamatergic neurotransmission has been demonstrated to have antidepressant effects in an inescapable stress rodent model (Trullas and Skolnick, 1990). Minimal data are available regarding the anxiolytic effects of L-Theanine (Lu et al., 2004). There is only one published study on the effects of L-Theanine on PTSD or regarding interactions of anxiolytics with L-Theanine that evaluated gene expression in the amygdala and hippocampus (Ceremuga et al., 2014).

Over the past three decades, herbal medications have gained popularity throughout the world (Hung and Ernst, 2010). Herbal use increased $380 \%$ in the U.S. between 1990 and 1997 nearly rivaling that of traditional medicine (Ang-Lee et al., 2001). Green tea is a popular herbal supplement, containing L-Theanine and has been found to decrease anxiety in the laboratory rat (Heese et al., 2009). Several laboratory studies have established and tested the Inescapable Tail-Shock Model of stress in rats. These tests have verified the neurobiological and behavioral alterations induced in the rat model and are similar to those found in human PTSD patients (Servatius et al., 1995; Garrick et al., 2001). The purpose of this study was to investigate the potential benefits of L-Theanine in the treatment of PTSD. Specifically, the research investigated the effects of L-Theanine, a component of green tea, on neurobehavior in a PTSD rodent model. This study provides data for the theoretical framework for the treatment of this condition and future studies (See Fig 1).

\section{Materials and Methods}

\section{Theoretical Model:}

After three days of restraint/shock, PTSD is induced in the rat model. PTSD leads to neurobehavioral changes demonstrated on the EPM, MWM, and FST. The

\section{Theoretical Framework}

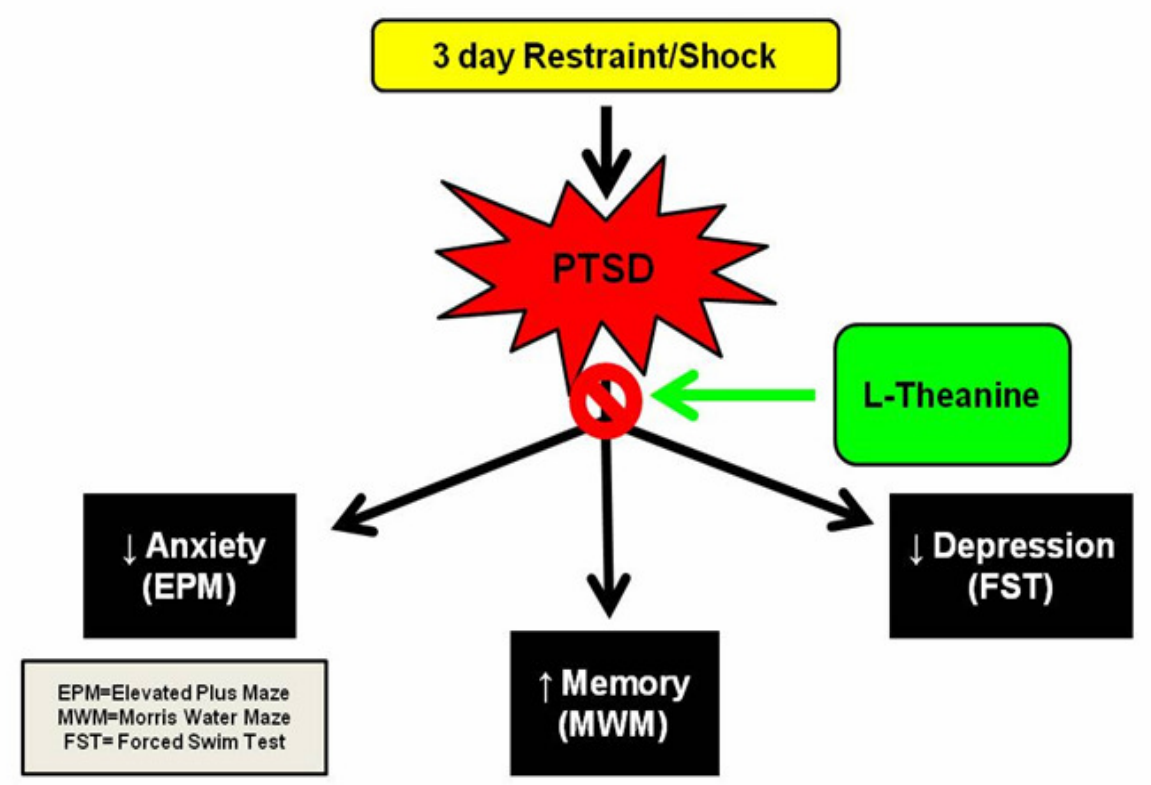

Fig 1. Theoretical Framework illustrating the relationship between traumatic event and development of PTSD symptoms effecting anxiety, memory, and depression. EPM = Elevated Plus Maze; MWM = Morris Water Maze; FST $=$ Forced Swim Test 
administration of L-Theanine to a subject with PTSD, it was hypothesized that the symptoms of anxiety, memory, and depression may be ameliorated (See Fig 1). Groups of male Sprague-Dawley rats were separated bedding. The animals went through a 7-day adaptation period in a temperature-controlled environment $\left(22 \pm 1^{\circ} \mathrm{C}, 60 \%\right.$ humidity) with a reverse light-dark cycle where they received 12 hours of light (6:00 PM to 6:00

Table 1. Overview of Experiment Design: Outlines the day by day progression of experiment design from day 1 to day 22 and tests completed on each day. PTSD=Post Traumatic Stress Disorder, EPM= Elevated Plus Maze, MWM= Morris Water Maze, FST= Forced Swim Test Random.

\begin{tabular}{|l|c|l|l|l|l|l|}
\hline & Acclimate & $\begin{array}{l}\text { Restraint/ } \\
\text { Shock } \\
\text { PT SD } \\
\text { Model }\end{array}$ & $\begin{array}{l}\text { Ten } \\
\text { Post- } \\
\text { Stress } \\
\text { Days }\end{array}$ & $\begin{array}{l}\text { MWM } \\
\text { Training }\end{array}$ & $\begin{array}{l}\text { MWM and } \\
\text { FST Training }\end{array}$ & $\begin{array}{l}\text { L-Theanine/Midazolam, Intervention } \\
\text { EPM, MWM, and FST Testing }\end{array}$ \\
\hline Days & $1-7$ & $\mathbf{8 - 1 0}$ & $\mathbf{1 1 - 1 9}$ & 20 & 21 & 22 \\
\hline Groups & $\begin{array}{c}\text { All rat } \\
\text { groups }\end{array}$ & $\begin{array}{c}\text { Stressed } \\
\text { (PTSD) rat } \\
\text { groups }\end{array}$ & $\begin{array}{l}\text { All rat } \\
\text { groups }\end{array}$ & $\begin{array}{l}\text { All rat } \\
\text { groups }\end{array}$ & All rat groups & \\
\hline
\end{tabular}

Table 2. Comparison of Symptoms of PTSD in Humans to Dysfunction Related to Stress in Rats (Restraint/shock) (Servatius et al., 1995; Garrick et al., 2001)

\begin{tabular}{|l|l|}
\hline \multicolumn{1}{|c|}{ PTSD in Humans } & Inescapable Tail-Shock Model of Stress in Rats (3 days) \\
\hline Weight loss & Suppressed feeding and body weight loss \\
\hline Difficulty falling or staying asleep, nightmares & Altered sleep patterns \\
\hline Psychomotor numbness & $\begin{array}{l}\text { Persistent behavioral abnormalities i.e. suppressed open-field } \\
\text { activity, longer hanging wire latencies }\end{array}$ \\
\hline Poor concentration; memory deficits & $\begin{array}{l}\text { Deficits in escape/avoidance, learning and learning of an } \\
\text { appetitive task }\end{array}$ \\
\hline Hyperarousal and/or exaggerated startle response & Exaggerated startle response \\
\hline
\end{tabular}

Table 3. Illustrates random assignment of 80 rats to eight groups. Four control and four PTSD with interventions

\begin{tabular}{|c|c|c|c|c|}
\hline Non-stressed & $\begin{array}{c}\text { Control (saline) } \\
\mathbf{n}=10\end{array}$ & $\begin{array}{c}\text { L-Theanine } \\
\mathbf{n}=10\end{array}$ & $\begin{array}{c}\text { Midazolam } \\
\mathbf{n}=10\end{array}$ & $\begin{array}{c}\text { L-Theanine + Midazolam } \\
\mathbf{n}=\mathbf{1 0}\end{array}$ \\
\hline $\begin{array}{l}\text { PTSD three day } \\
\text { Restraint/Shock }\end{array}$ & $\begin{array}{c}\text { Control (saline) } \\
\mathrm{n}=10\end{array}$ & $\begin{array}{c}\text { L-Theanine } \\
\mathrm{n}=10\end{array}$ & $\begin{array}{c}\text { Midazolam } \\
\mathrm{n}=10\end{array}$ & $\begin{array}{c}\text { L-Theanine }+ \text { Midazolam } \\
\mathrm{n}=10\end{array}$ \\
\hline
\end{tabular}

into PTSD and non-stressed groups. These groups were administered normal saline, a study drug, or a combination of study drugs to evaluate their effects on PTSD phenotypes. The Elevated Plus Maze was used to evaluate anxiety, Morris Water Maze for memory, and Forced Swim Test for depression phenotypes respectively. In the broader framework of our study, we attempted to refine this PTSD animal model.

\section{Post-Traumatic Stress Disease Induction Model:}

Eighty male Sprague-Dawley rats (Harlan Sprague-Dawley Laboratories) weighing 300-350 grams were used. They were housed in groups of three in polycarbonate "shoebox-sized" cages lined with
AM) and 12 hours of darkness (6:00 AM to 6:00 PM). The rats were allowed free access to food and water. The animals were handled for weighing, drug administration, and cleaning of cages only. After acclimation, the investigators measured body weight. Statistical analyses were conducted for body weight and rats were placed in groups to ensure equivalency regarding weight. The use of laboratory rats in this protocol was in accordance with the NIH Guide for the Care and Use of Laboratory Animals and received approval from the Institutional Animal Care and Use Committee at the Institute of Surgical Research, San Antonio, Texas. The design and timeline of this study is described in Table 1. 
Stress exposure consisted of a two hour immobilization and tail-shock session over three days. All sessions occurred between 8:00 AM and 12:00 PM in order to avoid disrupting normal sleep cycles. The animals were restrained in a Plexiglas tube, and 40 electric shocks (2mA, 3 seconds in duration) were applied at varying intervals (140-180 seconds). The timing and amplitude of shock were controlled by utilizing commercial hardware and software (Precision Animal Shocker, Coulbourn Instruments, Columbus, Ohio, USA). Rats were stressed for three consecutive days based on previous literature which indicated repeated stress sessions were more effective than a single stress session in producing physiological and behavioral abnormalities, including exaggerated acoustic startle reflex and reduced body weight (Servatius et al., 1995) (See Table 2).

Total of 80 rats were randomly assigned to the two groups as described in our previous work (Ceremuga et al., 2014). Forty rats were assigned to the nonstressed groups and forty were assigned to the 3-day-restraint shock PTSD rodent groups. There were four groups within the nonstressed rats (control vehicle, L-Theanine, midazolam, and L-Theanine + midazolam) and four groups in the stressed rats (control vehicle, L-Theanine, midazolam, and L-Theanine + midazolam). Each animal received a subcutaneous (SQ) injection of either: 1. control (saline); 2. L-Theanine (Sigma Chemical Co, St Louis, Missouri) $10 \mathrm{mg} / \mathrm{kg} ; 3$. midazolam (Versed $囚) 1.5 \mathrm{mg} / \mathrm{kg}$; or 4 . L-Theanine + midazolam (Versed $\AA$ ) $1.5 \mathrm{mg} / \mathrm{kg}$ (to evaluate interaction effects). The saline group acted as a control. All animals received equivalent volumes of subcutaneous injection to total $2 \mathrm{ml} / \mathrm{kg}$ (See Table 3).

All experimentation occurred on a timed schedule between 8:00 AM and 4:00 PM over four consecutive days to control for the circadian rhythm of the animals. Thirty minutes after the injection (group dependent) all rats were tested in the following sequence: EPM for five minutes, MWM for one minute, and then the FST for five minutes.

\section{Elevated Plus Maze:}

The EPM is an instrument utilized to measure anxiety in the rodent model (Lister, 1987; Falter et al., 1992; Treit et al., 1993; Shepherd et al., 1994; Rosa et al., 2000). This model has been validated in previous research studies (Montgomery, 1955; Pellow et al., 1985).

The EPM consists of two open arms and two closed arms 50 centimeters $(\mathrm{cm})$ in length and $10 \mathrm{~cm}$ in width. The open arms are directly opposite of each other, and similarly the closed arms are opposed one another. The open arm is covered with $1 \mathrm{~cm}$ high Plexiglas on the side, in order to prevent the rodent from falling. The maze was placed 50 centimeters above the floor. In addition to being constructed of wood, the interior floor was painted with waterproof epoxy paint in a dark color. This minimized rodent stimulation, and provided a waterproof surface when exposed to urine and feces. The elevated plus maze is shaped like a cross $(+)$, with the intersection of the four arms measuring 10 x $10 \mathrm{~cm}$ (Pellow et al., 1985).

All rats were injected subcutaneously 30 minutes before behavioral evaluation with the EPM as previous studies demonstrated an anxiolytic effect between 20-30 minutes. Between the time of injection and testing, the rat was placed back into its cage in order to reduce any confounding influences or exposure to any additional unfamiliar environments. A testing period of five minutes was employed on the EPM. Each rat was placed in the center of the EPM facing an open arm. During the 5-minute test, the number of entries into each type of arm and the time spent in each arm was scored. A rat was considered to have entered an arm via the ANY-maze software. At the end of the test, the number of entries into the open arms was expressed as a percentage of the total number of arm entries. The time spent on the open arms was also expressed as a percentage of the time spent on both the open and the closed arms. An increase in the percentage of time spent in the open arms reflected an anxiolytic effect. Between testing each animal, the EPM was cleansed with nonfragrant soap and water and dried. Each experimental session was recorded for five minutes by the ANY-maze software and validated by videotapes analyzed by an investigator blinded to the treatment received by each animal that underwent testing.

\section{Morris Water Maze:}

Specific aspects of spatial memory have been extensively studied using the Morris Water Maze. Animals have evolved an optimal strategy to explore their environment and escape from water using minimal effort. After previous exposure to the water maze setup and using only available external cues, the time it takes a rat to find a hidden platform in a pool of water is a measure of spatial memory (Wenk, 2004). With minor modifications, all animals were exposed to the two-day water maze test as previously described (Frick et al., 2000). In order to evaluate learning and memory in rodents, the water maze test was utilized. The setup consisted of a circular $(190 \mathrm{~cm}$ in diameter $)$ water-filled tank (up to $30 \mathrm{~cm}$ deep; temperature: $22 \pm 2{ }^{\circ} \mathrm{C}$ ) that was used for the test. A non-toxic dye was used to make the water opaque. The tank was divided into four zones. A platform $(18 \mathrm{~cm} \times 18 \mathrm{~cm})$ was submerged $2 \mathrm{~cm}$ below the water surface, in Zone 3 . The test was conducted in a small room where 
extra-maze cues were kept consistent throughout the experiment. An overhead camera was connected to a computer to record the data. Over two days, all the rats were exposed to the setup six times, for training purposes. Each rat was given a total of 60 seconds to randomly explore the water maze and find the platform by chance. If the rat did not find the platform by chance, guidance was provided. The rat was then allowed ten seconds on the platform to familiarize itself to the location, relative to the available visual clues in the room. Each swim started from a designated position to maintain consistency for each rat. A 30-minute interval separated three blocks of six swims. Data recording was only initiated after the training period. The probe trial confirmed any improvements in spatial memory and learning. The data recorded included distance, mean speed, rotations, entries, time, latency to first entry and platform entries. The probe trial involved removing the platform and a single trial of 60 seconds was performed. The percentage of time spent in each zone was recorded. A sample trial where the rat swims

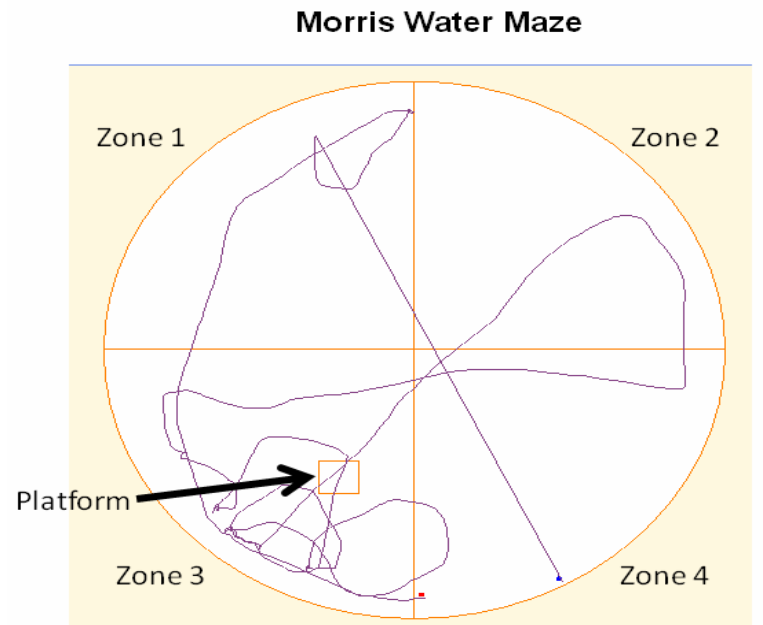

Fig 2. Morris Water Maze map, as demonstrated from ANY-maze ${ }^{\circledR}$ software. Zone 3 is the area where platform was located, and illustrates the rat searching for the platform

in each zone of the Morris Water Maze and Zone 3, where the platform was located, is shown in Fig 2.

\section{Forced Swim Test:}

After evaluation in the Morris Water Maze, animals were carried to a separate room and evaluated in the Behavioral Despair Test (also called Forced Swim Test or Porsolt test). Since its development, the Forced Swim Test has been a valid assessment of depression in the rodent model (Porsolt et al., 1977; Porsolt et al., 1978; Castagne et al., 2011).
In two sessions separated by 24 hours, rats were forced to swim in a narrow cylinder from which they cannot escape. The transparent cylinders $(20 \mathrm{~cm}$ diameter $\times 40 \mathrm{~cm}$ high) contained water $\left(25 \pm 2^{\circ} \mathrm{C}\right)$ to a depth of $13 \mathrm{~cm}$, and the water was changed for each animal. The first session, lasting 15 minutes, was the training session and was conducted 24 hours prior to drug administration and without behavioral recording. After 15 minutes, the animal was removed, dried and placed in its cage. This training session acclimated the rats to the test situation, thereby providing a stable, high level of immobile behavior during the 5-minute test session 24 hours later that was video recorded. Three trained research assistants blindly reviewed videotapes, and the amount of time the rats were immobile was calculated.

The measurement of the duration of immobility when rodents are exposed to an inescapable situation (Forced Swim Test) is a reflection of depression. In this model of depression, the longer the duration of immobility, the greater the behavioral despair or depression. The amount of immobility time was statistically analyzed between groups.

\section{Results}

Data regarding weight gain between the 40 control (non-stressed) and 40 PTSD (stressed) rats were significantly different $(p<.001)$, where the control rats gained an average of 55.4 grams compared to 37.4 grams for the PTSD rats over the 10 post stress days. All neurobehavior data were analyzed using a two-tailed multivariate analysis of variance (MANOVA). A determination of statistical significance in neurobehavior using L-Theanine in a rodent model was made. If significance was found, a LSD post hoc was used.

\section{Elevated Plus Maze:}

Analysis of the ratio open arm time to total time on EPM revealed a statistically significant difference between the control midazolam and PTSD control group ( $p=0.004)$. For the one-way ANOVA, significant between group differences were obtained: $F(7,72)$ $=2.62, p=0.018, \eta^{2}=0.203(20.3 \%$ of the variability in the outcome was attributable to between-group differences). The $\mathrm{C}-\mathrm{M}$ group had the highest mean $(M=$ $36.04)$ and the P-V group had the lowest $(M=10.52)$. Given the homogeneity of variance assumption was not met $(p=0.004)$, the Games-Howell MCP was performed and no pairwise comparisons were significant. Given outliers and/or slight non-normality of the distribution, the investigators used the Kruskal-Wallis test and, as with the ANOVA, significance was obtained: $\chi^{2}(7)=$ $22.65, p=0.002$. Moreover, a square root transformation did improve the distribution of this outcome, and 
Table 4. Open Arm Entries \& Ratio of Open Arm Time in groups of rats in the Elevated Plus Maze, $\mathrm{SEM}=$ Standard Error of the Mean

\begin{tabular}{|l|c|c|c|c|c|}
\hline Group & $\begin{array}{l}\text { Sample } \\
\text { Size }\end{array}$ & $\begin{array}{l}\text { Mean Open } \\
\text { Arm Entries }\end{array}$ & $\begin{array}{l}\text { Open Arm } \\
\text { Entries SEM }\end{array}$ & $\begin{array}{l}\text { Mean Ratio } \\
\text { Open Arm } \\
\text { /Total Time }\end{array}$ & $\begin{array}{l}\text { Ratio Open Arm } \\
\text { Total Time SEM }\end{array}$ \\
\hline Control Vehicle & 10 & 8.00 & 1.23 & 12.83 & 2.39 \\
\hline Control L-Theanine & 10 & 10.00 & 1.63 & 12.61 & 2.36 \\
\hline Control Midazolam & 10 & 4.40 & 0.96 & 36.04 & 9.76 \\
\hline $\begin{array}{l}\text { Control Midazolam } \\
\text { + L-Theanine }\end{array}$ & 10 & 7.60 & 1.90 & 15.42 & 6.85 \\
\hline PTSD Vehicle & 10 & 6.70 & 1.25 & 10.52 & 1.64 \\
\hline PTSD L-Theanine & 10 & 16.10 & 1.85 & 17.53 & 2.41 \\
\hline PTSD Midazolam & 10 & 6.70 & 1.23 & 28.84 & 8.75 \\
\hline $\begin{array}{l}\text { PTSD Midazolam }+ \\
\text { L-Theanine }\end{array}$ & 10 & 10.60 & 2.12 & 27.84 & 5.66 \\
\hline
\end{tabular}

\section{Ratio Open-Arm Time to Total Time on Elevated Plus Maze}

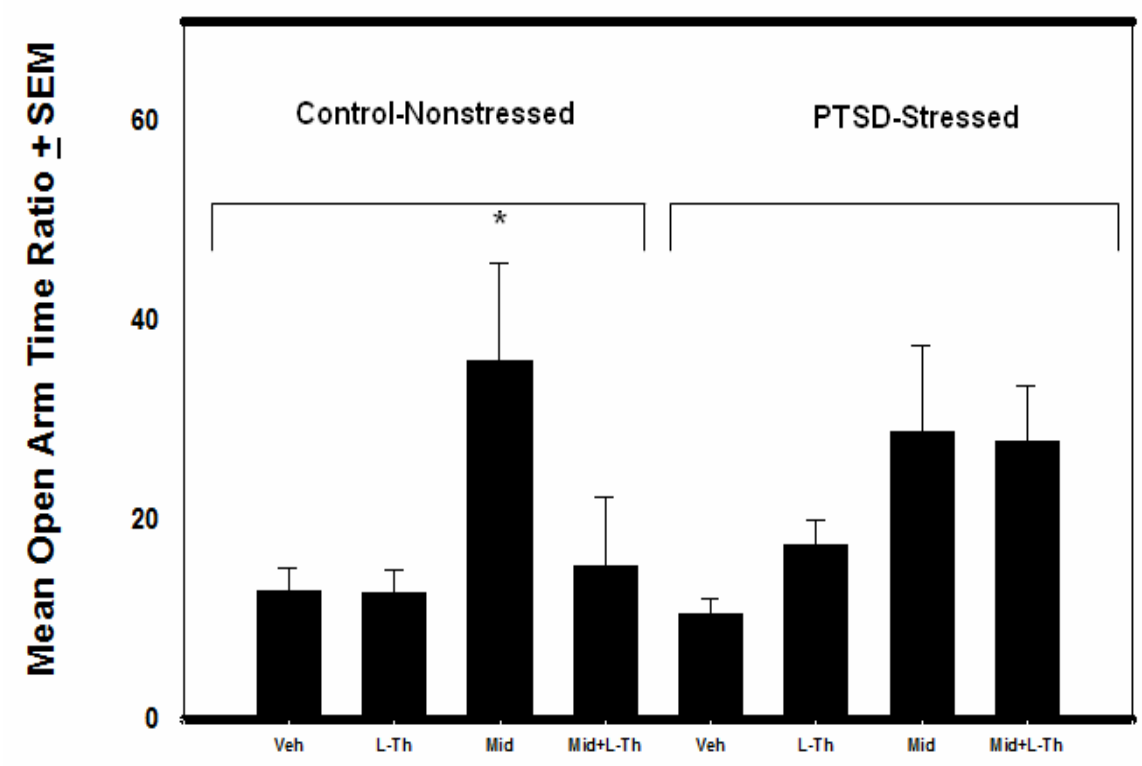

Treatment Groups

Fig 3. Ratio Open-Arm Time to Total Time on Elevated Plus Maze shows the calculated ratio of time that the rat spent on the open arm compared to the total time on the maze. X axis is treatment groups and Y axis is mean open arm time ratio in seconds. Veh=Vehicle, $\mathrm{L}-\mathrm{Th}=\mathrm{L}$-Theanine, Mid=Midazolam, Mid+L- Th= Midazolam and L-Theanine, SEM=Standard Error of the Mean.

significance was still obtained per the ANOVA: $F(7,72)$ $=3.32, p=0.004, \eta^{2}=0.244$ and per the Tukey post hoc test, there is one pairwise significant comparison, that being CM (higher mean) vs. PV (See Fig 3, 4 and Table 4).

\section{Morris Water Maze:}

In the MWM test, using a one-way ANOVA, there were no statistical significant differences found between groups when looking at distance, mean speed, 
and entries to the platform area (Zone 3) (See Table 5 and Fig 5).

\section{Forced Swim Test:}

For the one-way ANOVA, significant between group differences were found: $F(7,71)=2.51, p=0.023, \eta^{2}=$ $0.198(19.8 \%$ of the variability in the outcome was attributable to between-group differences). The P-M group had the highest mean $(M=127.7$ and the $\mathrm{C}-\mathrm{M}+\mathrm{L}$ group had the lowest $(M=54.93)$ and per the Games-Howell post hoc test (given heterogeneous variances) none of the pairwise comparisons were significant (See Fig 6).

\section{Discussion}

The psychological, physical and emotional symptoms of PTSD are harmful and complex, including intrusive thoughts, nightmares, anxiety, avoidance, or reminders of the traumatic event, and hyperarousal (Ursano et al., 2009). Depression, anxiety, panic, and substance abuse are used as key symptoms in differentiating between the specific and general factors of PTSD (Gootzeit and Markon, 2011). An optimal treatment modality has not been found. Current treatments for PTSD are multimodal, complicated, inconsistent, and far from understood. This was the first study evaluating L-Theanine in the treatment of PTSD.

The administration of L-Theanine to a rodent with induced PTSD to ameliorate symptoms of anxiety,
The PTSD induction model in which rats were exposed to a two hour immobilization and tail-shock session over three days was validated in our study. This was evidenced by the significantly different weight gain between the control and PTSD groups $(p<.001)$. It has been demonstrated that there is direct correlation with decreased food intake and statistically significant weight loss in animals with PTSD (Servatius et al., 1995; Pulliam et al., 2010) Additionally, statistically significant results were found between the non-stressed group and three day restraint shock PTSD rodent model group in the EPM. Analysis of the ratio open arm time to total time on EPM revealed a statistically significant difference between the control midazolam and PTSD control group ( $p=0.004)$. Furthermore, although not statistically significant, the control vehicle group had a greater open arm time ratio (12.83) compared to the PTSD vehicle group (10.52), a trend potentially indicating less anxiety in the control group.

L-Theanine is a component of green tea and has gained recent popularity for the self-treatment of anxiety; recent animal studies have shown anxiety to be decreased significantly when L-Theanine is combined with midazolam $(p=0.005)$ (Heese et al., 2009). We evaluated the effects of L-Theanine alone and combined with midazolam to alleviate PTSD phenotypes utilizing the EPM, MWM, and FST in Sprague-Dawley rats. Overall, evaluation of the data

Table 5. Morris Water Maze time spent by each group of rats in Zone 3 (platform location), SEM=Standard Error of the Mean

\begin{tabular}{|l|l|l|l|}
\hline Group & Sample Size & Mean Time in Zone 3 & SEM \\
\hline Control Vehicle & 10 & 16.370 & 1.08 \\
\hline Control L-Theanine & 10 & 18.830 & 1.23 \\
\hline Control Midazolam & 10 & 17.230 & 1.89 \\
\hline Control Midazolam + L-Theanine & 10 & 16.320 & 1.89 \\
\hline PTSD Vehicle & 10 & 16.290 & 1.99 \\
\hline PTSD L-Theanine & 10 & 16.680 & 1.26 \\
\hline PTSD Midazolam & 10 & 17.970 & 2.39 \\
\hline PTSD Midazolam + L-Theanine & 10 & 15.670 & 1.09 \\
\hline
\end{tabular}

memory, and depression was studied. Rats were separated into PTSD and non-stressed groups and administered various pharmacological interventions. The following phenotypes were measured as follows: The Elevated Plus Maze was used to evaluate anxiety, Morris Water Maze for memory, and Forced Swim Test for depression. collected in this study did not support our hypothesis that L-Theanine may ameliorate the symptoms of PTSD. However, our theoretical framework and experimental model was validated through our implemented controls. 


\section{Open Arm Entries on Elevated Plus Maze}

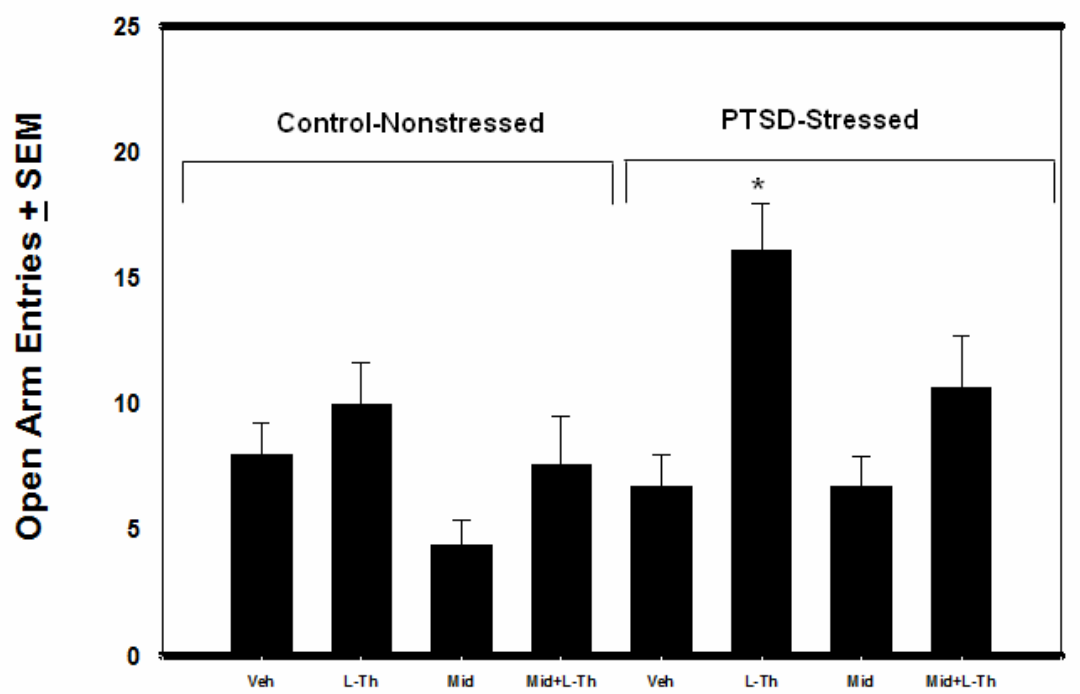

Treatment Groups

Fig 4. Open-Arm Entries on Elevated Plus Maze shows the number of times the rat ventured onto the open arm on the elevated plus maze. $\mathrm{X}$ axis is treatment groups and $\mathrm{Y}$ axis is mean open arm time in seconds. Veh=Vehicle, L-Th= L-Theanine, Mid=Midazolam, Mid+L-Th=Midazolam and L-Theanine, SEM=Standard Error of the Mean.

\section{Mean TIme in Zone 3 in the Morris Water Maze}

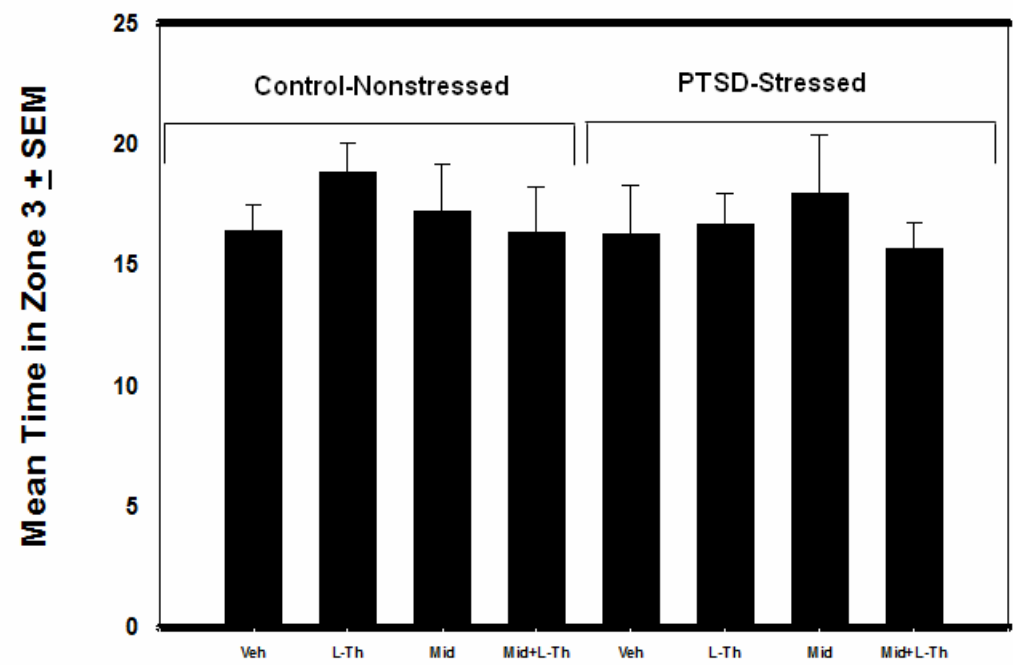

Treatment Groups

Fig 5. Mean time in Zone 3 in the Morris Water Maze. $X$ axis is treatment groups and $Y$ axis is mean open arm time in seconds. Veh=Vehicle, L-Th= L-Theanine, Mid=Midazolam, Mid+L-Th=Midazolam and L-Theanine, SEM=Standard Error of the Mean.

The EPM was used to evaluate the amount of anxiety-like behavior experienced by the rat (Lister, 1987; Falter et al., 1992; Treit et al., 1993; Shepherd et al., 1994; Rosa et al., 2000). During the 5-minute test, the number of entries into the open or closed arms, as well as the time spent in each arm, was a measure of anxiety-like behavior. In previous studies, L-Theanine has been shown to decrease anxiety (Heese et al., 
2009). Our results showed that the PTSD L-Theanine group had the higher mean $(M=16.10)$ entries into the open arm compared to all other groups. The control midazolam group had the lowest number of entries $(M=4.4)$. Although the addition of L-Theanine did not result in any significant difference between the groups, these data may suggest that L-Theanine administered to the PTSD rats may have a positive effect in decreasing the aversion of entering the open arm.

The MWM was used to investigate learning and memory in rats (Wenk, 2004). The time it takes the rats to find the hidden platform is the determinate of spatial memory. Our data showed no significance with the use of L-Theanine, when pertaining to distance, mean speed, and entries into the Zone 3 where the platform was located.

The FST was used to evaluate the depressive phenotype of rats (Porsolt et al., 1977; Porsolt et al., 1978; Castagne et al., 2011). In the FST, longer duration of immobility reflects greater behavioral despair or depression. According to our data, there was a significant difference in the mean movement time between our control and experimental groups. Of all groups, the PTSD induced animals who were administered midazolam had the highest mean movement time, reflecting a decrease in depressive phenotype. The control group that was administered L-Theanine and midazolam had the lowest mean movement time, reflecting greatest amount of behavioral despair or depression. With regards to L-Theanine, our results did not support our hypothesis. A one-time dose with L-Theanine $10 \mathrm{mg} / \mathrm{kg}$ or in combination with midazolam (Versed $囚$ ) $1.5 \mathrm{mg} / \mathrm{kg}$ was insufficient in providing a significant decrease in depression as evaluated by the FST.

This was the first study evaluating L-Theanine in the treatment of PTSD. Future investigations may take into account the total amount of L-Theanine administered (10 $\mathrm{mg} / \mathrm{kg}$ in this experiment). Routes of administration may also be considered. An intraperitoneal administration of $10 \mathrm{mg} / \mathrm{kg}$ as was utilized in our study based on previous research (Sugiyama and Sadzuka, 1999). Other studies (Yokogoshi et al., 1998; Yokogoshi and Terashima, 2000) have suggested that intragastric and direct brain administration of L-Theanine may also be effective in the rat model.

The timing of administration, such as multidose or prophylaxis, may yield different results in preventing or aborting PTSD symptomology. It is known that some treatments of PTSD (i.e. antidepressants) may require an extended period of time to affect neurobehavior (Gelenberg and Chesen, 2000; Dremencov et al., 2004).
As with SSRIs, it may take at least 1 week for neurobehavioral changes to be evident (Taylor et al., 2006). Future studies of L-Theanine may utilize our current validated model with an extended dosing period to obtain steady state for the period of time needed to alter neurobiology. The meticulous study of the neurobiological effects of L-Theanine may further define the mechanism of action of L-Theanine, enabling future research to be more focused.

\section{Conclusions}

In conclusion, PTSD is a devastating neuropsychological injury with a multitude of symptoms including: increased anxiety, hypervigilance, impaired memory and depression. Through use of the Restraint Tail-Shock device, PTSD was induced in 40 Sprague-Dawley rats. After adequate time to develop symptomatology, depending upon group assignment, rats were given a pharmacological intervention including L-Theanine. The rats were then subjected to a series of neurobehavioral testing in order to evaluate anxiety (EPM), spatial memory (MWM) and depression (FST). Our results showed no significant difference between rats with single dose intraperitoneal L-Theanine $10 \mathrm{mg} / \mathrm{kg}$ and in combination with Midazolam $1.5 \mathrm{mg} / \mathrm{kg}$ and control vehicle. However, the theoretic framework and PTSD Disease Induction Model were validated based on this research. This model will be useful in future research applications.

\section{Acknowledgements}

The researchers would like to acknowledge their families and the support they provided, as well as the US Army Graduate Program in Anesthesia Nursing faculty for their expertise and guidance. We would also like to thank Dr. Dale Glaser for his invaluable assistance with processing the data and the US Army Institute of Surgical Research for allowing us to conduct this research at their facility. This study was funded by the TriService Nursing Research Program.

\section{References}

Ang-Lee, M. K., J. Moss, and C. S. Yuan. 2001. Herbal medicines and perioperative care. Jama 286: 208-216

Brewin, C., J. S. Kleiner, J. J. Vasterling, and A. P. Field. 2007. Memory for emotionally neutral information in posttraumatic stress disorder: A meta-analytic investigation. Journal of Abnormal Psychology 116: 448

Castagne, V., P. Moser, S. Roux, and R. D. Porsolt. 2011. Rodent models of depression: forced swim and tail suspension behavioral despair tests in rats and mice. Curr Protoc Neurosci Chapter 8: Unit 8 10A. doi: 10.1002/0471142301.ns0810as55

Ceremuga, T. E., S. Martinson, J. Washington, R. Revels, J. Wojcicki, D. Crawford, R. Edwards, J. L. Kemper, W. L. Townsend, G. M. Herron, G. A. Ceremuga, G. Padron, 
and M. Bentley. 2014. Effects of L-theanine on posttraumatic stress disorder induced changes in rat brain gene expression. ScientificWorldJournal 2014: 419032. doi: 10.1155/2014/419032

Charney, D. S., S. J. Mihic, and R. A. Harris. 2001. Chapter 17: Hypnotics and Sedatives. In: Goodman and Gilman's The Pharmacological Basis of Therapeutics. J. G. Hardman and L. E. Limbird, Eds. McGraw-Hill, New York. p. 399-427.

Dremencov, E., I. Gispan-Herman, M. Rosenstein, A. Mendelman, D. H. Overstreet, J. Zohar, and G. Yadid. 2004. The serotonin-dopamine interaction is critical for fast-onset action of antidepressant treatment: in vivo studies in an animal model of depression. Progress in Neuro-Psychopharmacology and Biological Psychiatry 28: 141-147

Eschenauer, G., and B. V. Sweet. 2006. Pharmacology and therapeutic uses of theanine. Am J Health Syst Pharm 63: 26, 28-30. doi: 10.2146/ajhp050148

Falter, U., A. J. Gower, and J. Gobert. 1992. Resistance of baseline activity in the elevated plus-maze to exogenous influences. Behav Pharmacol 3: 123-128

Frick, K. M., E. T. Stillner, and J. Berger-Sweeney. 2000. Mice are not little rats: species differences in a one-day water maze task. Neuroreport 11: 3461-3465

Garrick, T., N. Morrow, A. Y. Shalev, and S. Eth. 2001. Stress-induced enhancement of auditory startle: an animal model of posttraumatic stress disorder. Psychiatry 64: 346-354

Gelenberg, A. J., and C. L. Chesen. 2000. How fast are antidepressants? J Clin Psychiatry 61: 712-721

Gootzeit, J., and K. Markon. 2011. Factors of PTSD: differential specificity and external correlates. Clin Psychol Rev 31: 993-1003. doi: 10.1016/j.cpr.2011.06.005

Heese, T., J. Jenkinson, C. Love, R. Milam, L. Perkins, C. Adams, S. McCall, and T. E. Ceremuga. 2009. Anxiolytic effects of L-theanine--a component of green tea--when combined with midazolam, in the male Sprague-Dawley rat. Aana J 77: 445-449

Hung, S. K., and E. Ernst. 2010. Herbal medicine: an overview of the literature from three decades. J Diet Suppl 7: 217-226. doi: 10.3109/19390211.2010.487818

Jankowsi, K. 2010. PTSD and Physical Health. National Center for PTSD.

Juneja, L. R., D.-C. Chu, T. Okubo, Y. Nagato, and H. Yokogoshi. 1999. L-Theanine- a unique amino acid of green tea and its relaxation effect in humans. Trends in Food Science \& Technology 10: 199-204

Lister, R. G. 1987. The use of a plus-maze to measure anxiety in the mouse. Psychopharmacology (Berl) 92: 180-185

Lu, K., M. A. Gray, C. Oliver, D. T. Liley, B. J. Harrison, C. F. Bartholomeusz, K. L. Phan, and P. J. Nathan. 2004. The acute effects of L-theanine in comparison with alprazolam on anticipatory anxiety in humans. Hum Psychopharmacol 19: 457-465

Montgomery, K. C. 1955. The relation between fear induced by novel stimulation and exploratory behavior. J Comp Physiol Psychol 48: 254-260

Pellow, S., P. Chopin, S. E. File, and M. Briley. 1985. Validation of open:closed arm entries in an elevated plus-maze as a measure of anxiety in the rat. J Neurosci Methods 14: 149-167

Porsolt, R. D., A. Bertin, and M. Jalfre. 1978. "Behavioural despair" in rats and mice: strain differences and the effects of imipramine. Eur J Pharmacol 51: 291-294
Porsolt, R. D., M. Le Pichon, and M. Jalfre. 1977. Depression: a new animal model sensitive to antidepressant treatments. Nature 266: 730-732

Pulliam, J. V., A. M. Dawaghreh, E. Alema-Mensah, and P. M. Plotsky. 2010. Social defeat stress produces prolonged alterations in acoustic startle and body weight gain in male Long Evans rats. J Psychiatr Res 44: 106-111. doi: 10.1016/j.jpsychires.2009.05.005

Roan, S. 2009. L-Theanine comes into focus. Los Angeles Times.

Rosa, V. P., N. Vandresen, A. V. Calixto, D. F. Kovaleski, and M. S. Faria. 2000. Temporal analysis of the rat's behavior in the plus-maze: effect of midazolam. Pharmacol Biochem Behav 67: 177-182

Servatius, R. J., J. E. Ottenweller, and B. H. Natelson. 1995. Delayed startle sensitization distinguishes rats exposed to one or three stress sessions: further evidence toward an animal model of PTSD. Biol Psychiatry 38: 539-546

Shepherd, J. K., S. S. Grewal, A. Fletcher, D. J. Bill, and C. T. Dourish. 1994. Behavioural and pharmacological characterisation of the elevated "zero-maze" as an animal model of anxiety. Psychopharmacology (Berl) 116: 56-64

Shiner, B., R. E. Drake, B. V. Watts, R. A. Desai, and P. P. Schnurr. 2012. Access to VA services for returning veterans with PTSD. Mil Med 177: 814-822

Sugiyama, T., and Y. Sadzuka. 1999. Combination of theanine with doxorubicin inhibits hepatic metastasis of M5076 ovarian sarcoma. Clin Cancer Res 5: 413-416

Taylor, M. J., N. Freemantle, J. R. Geddes, and Z. Bhagwagar. 2006. Early onset of selective serotonin reuptake inhibitor antidepressant action: systematic review and meta-analysis. Arch Gen Psychiatry 63: 1217-1223

Treit, D., J. Menard, and C. Royan. 1993. Anxiogenic stimuli in the elevated plus-maze. Pharmacol Biochem Behav 44: 463-469

Trullas, R., and P. Skolnick. 1990. Functional antagonists at the NMDA receptor complex exhibit antidepressant actions. Eur J Pharmacol 185: 1-10

Ursano, R. J., L. Zhang, H. Li, L. Johnson, J. Carlton, C. S. Fullerton, and D. M. Benedek. 2009. PTSD and traumatic stress from gene to community and bench to bedside. Brain Res 1293: 2-12

Wenk, G. L. 2004. Assessment of spatial memory using the radial arm maze and Morris water maze. Curr Protoc Neurosci Chapter 8: Unit 8 5A. doi: 10.1002/0471142301.ns0805as26

Yokogoshi, H., M. Mochizuki, and K. Saitoh. 1998. Theanine-induced reduction of brain serotonin concentration in rats. Biosci Biotechnol Biochem 62: 816-817

Yokogoshi, H., and T. Terashima. 2000. Effect of theanine, r-glutamylethylamide, on brain monoamines, striatal dopamine release and some kinds of behavior in rats. Nutrition 16: 776-777 